\title{
COMPUTATION OF SUPERCONDUCTIVITY IN THIN FILMS
}

\author{
By \\ San Yih Lin \\ and \\ Yisong Yang
}

IMA Preprint Series \# 541

June 1989 


\title{
COMPUTATION OF SUPERCONDUCTIVITY IN THIN FILMS*
}

\author{
SAN YIH LIN† AND YISONG YANG
}

\begin{abstract}
This paper presents a method for computing the physical states in superconducting thin films under the influence of a parallel uniform external magnetic field. The governing equations are the Ginzburg-Landau equations which in general possess multiple solutions and the physical states are those which minimize the total energy. In our approach the energy functional is used to generate a gradient flow and the physical states are obtained in the large time limit. The numerical results completely verify the Meissner effect and the fine structure of the solutions exhibits the occurrence of a symmetric nucleation of superconductivity at intermediate fields.
\end{abstract}

AMS(MOS) subject classifications. 65M10, 81J05

1. Introduction. Slightly below the transition temperatures, the behaviour of superconducting materials is governed by the Ginzburg-Landau (GL) differential equations [11]. Although these equations were originally introduced phenomenologically, Gorkov [12] was able to derive them theoretically from his formulation of the Bardeen-Cooper-Schrieffer (BCS) theory where the microscopic structure of a superconductor is replaced by a complex scalar field $\phi$ which is an order parameter representing the density of superconducting electron pairs and interacting with the excited electromagnetic vector potential $\mathbf{A}$. It is more often the GL equations, rather than the BCS theory itself, which have led technological advances, since they allow practical calculations for various samples. However, on the other hand, except for some extreme cases, the exact solutions of the GL equations are difficult to obtain due to the complexity of the system and, in general, suitable approximation methods must be resorted to. In particular, perturbative analysis has shown that the destruction of superconductivity in thin films by a magnetic field exceeding a critical external field strength which increases with decreasing thickness of the films [11] and that there exists a third critical field in type II superconductivity, so that, at which, a thin superconducting layer appears on the surface of the film [19]; a shooting method has been employed to find the properties of a class of solutions to the thin film GL equations [16]; a direct variational method has been used to study the interactions of superconducting vortices in the absence of an external field $[15,18]$; and, more recently, the Monte Carlo simulation has widely been applied to the lattice versions of the models in two, three, and four dimensions $[6,7,13,14]$. All of these are meant to compute the stationary or minimization points of the total energy $E(\phi, \mathbf{A})$.

*Supported by Institute for Mathematics and its Applications, and Supercomputer Institute, University of Minnesota

†Institute of Aeronautics and Astronautics, National Chen Kung University, Tainan, Taiwan 70101, R. O. C.

$\ddagger$ Institute for Mathematics and its Applications, University of Minnesota, Minneapolis, MN 55455, USA 
In this paper, we take a new approach to the above problem. Roughly speaking, we will find the stationary points of $E(\phi, \mathbf{A})$ in the $t \rightarrow \infty$ limit of the gradient (or heat) flow $(\phi(t), \mathbf{A}(t))$ generated by the equation $\left(\phi_{t}, \mathbf{A}_{t}\right)=-\delta E(\phi, \mathbf{A})$ where $\delta$ denotes the Fréchet differentiation. Methods of this nature have extensively been used earlier, for example, in the study of the existence of harmonic maps $[9,10]$. The purpose of the present paper is to focus exclusively on computing the solutions of the one-dimensional thin film GL equations. It is hoped that our method here may still be explored further to apply to the models in higher dimensions.

We will consider a superconducting film of thickness $2 \ell$ in a parallel (or tangential) constant magnetic field $H$. In normalized units and reduced variables, the GL equations are $[5,17]$ :

$$
\left\{\begin{array}{l}
\phi^{\prime \prime}(x)=\lambda \phi\left(\phi^{2}-1\right)+\lambda H^{2} A^{2} \phi \\
A^{\prime \prime}(x)=\phi^{2} A, \quad-\ell<x<\ell \\
\phi^{\prime}( \pm \ell)=0, \quad A^{\prime}( \pm \ell)=1
\end{array}\right.
$$

where, now, $\phi$ and $H A$ are real scalar fields representing the order parameter and the electromagnetic potential respectively, $\lambda>0$ is a dimensionless coupling constant so that $\lambda<1 / 2$ or $>1 / 2$ characterizes type I or II superconductors, and the excited magnetic field in the film is given by $B(x)=H A^{\prime}(x)$. Eqs.(1.1) are the Euler-Lagrange equations of the total energy (cf. [5])

$$
E(\phi, A)=\frac{1}{2} \int_{-\ell}^{\ell} d x\left\{\left(\phi^{\prime}\right)^{2}+\lambda H^{2}\left(\left[A^{\prime}-1\right]^{2}+A^{2} \phi^{2}\right)+\frac{\lambda}{2}\left(\phi^{2}-1\right)^{2}\right\} .
$$

In general, Eqs.(1.1) may have multiple solutions at different energy levels and the real physical states are those with the least energy value.

If $H=0$, the only energy minimizers are

$$
\phi(x)= \pm 1, \quad A(x)=\sinh x / \cosh \ell .
$$

Our purpose is to compute the energy minimizers at an arbitrary value $H=H_{0} \neq 0$. The method is as follows.

Define a time-dependent external field $H(t) \in C[0, \infty)$ connecting the values $H=0$ and $H=H_{0}$, i.e., $H(0)=0$ and $H(t) \equiv H_{0}$ for $t \geq$ some $t_{0}>0$. Insert $H=H(t)$ into the energy $E(\phi, A)$ and switch on the time-dependence of $(\phi, A)$ according to the convention as mentioned before:

$$
\left(\phi_{t}, A_{t}\right)=-\delta E(\phi, A)
$$


The equation (1.3) leads to an initial value problem

$$
\left\{\begin{array}{l}
\phi_{t}=\phi_{x x}-\lambda \phi\left(\phi^{2}-1\right)-\lambda H^{2}(t) A^{2} \phi, \\
A_{t}=A_{x x}-\phi^{2} A, \quad-\ell<x<\ell, \quad t>0 ; \\
\phi_{x}( \pm \ell, t)=0, \quad A_{x}( \pm \ell, t)=1, \quad t>0, \\
\phi=\phi_{0}(x), \quad A=A_{0}(x), \quad-\ell<x<\ell, \quad t=0 .
\end{array}\right.
$$

If the initial data $\left(\phi_{0}, A_{0}\right)$ are chosen to be physical, namely, $\left(\phi_{0}, A_{0}\right)$ satisfies (1.2), then (1.3) or (1.4) determines the time-evolution of the field configurations in a superconducting film (cf. e.g. [2]). It is not hard to prove that [20], as $t \rightarrow \infty$, the solutions of (1.4) tend to those of (1.1) which should be the desired least energy solutions at $H=H_{0}$ by the consistency of the time-evolution equation (1.3) and the least action principle, although a mathematically rigorous justification has not been worked out yet.

In this paper we will integrate Eqs.(1.4) numerically by the backward (implicit) finite difference scheme. Analysis shows that the convergence of the semi-discretized equations is of the second order and that of the backward difference scheme the first order in time and second order in space. A series of numerical results are presented. These results indicate that at small or large external fields, the Meissner effect is complete, that is, the superconducting films behave either like diamagnetic bulk materials or like normal conductors; at intermediate external fields, there is a symmetric nucleation of superconductivity in the middle of the films. For large values of $\lambda>0$, completely superconducting and normal regions co-exist and, as $\lambda \rightarrow \infty$, the superconducting core can be squeezed into an arbitarily narrow region which implies that a nonlinear desingularization phenomenon [4] occurs in a finite one-dimensional sample in analogy with that in two-dimensions [1,3]. This work shows that the gradient flow method may provide us a very efficient and powerful computational tool in calculating the thin film superconductivity and Eq.(1.3) can indeed yield a correct description of the time-development of the physical solutions of the problem.

2. The finite difference scheme. In order to have a suitable function space setting, we introduce a translation

$$
\mathcal{A}=A-x, \quad \mathcal{A}_{0}=A_{0}-x .
$$

Now Eqs.(1.4) become

$$
\left\{\begin{array}{l}
\phi_{t}=\phi_{x x}-\lambda \phi\left(\phi^{2}-1\right)-\lambda H^{2}(t)(\mathcal{A}+x)^{2} \phi, \\
\mathcal{A}_{t}=\mathcal{A}_{x x}-\phi^{2}(\mathcal{A}+x), \quad-\ell<x<\ell, \quad t>0 \\
\phi_{x}( \pm \ell, t)=\mathcal{A}_{x}( \pm \ell, t)=0, \quad t>0, \\
\phi=\phi_{0}, \quad \mathcal{A}=\mathcal{A}_{0}, \quad-\ell<x<\ell, \quad t=0 .
\end{array}\right.
$$

In what follows, we shall use the notation $D^{k}=\partial^{k} / \partial x^{k}, H^{k}(-\ell, \ell)=W^{k, 2}(-\ell, \ell)$ and denote the norms of $H^{k}(-\ell, \ell)$ and $C^{k}[-\ell, \ell]$ by \|\|$_{k}$ and ||$_{k}(k=0,1,2, \cdots)$, respectively. 
It has been shown in [20] that, for any $\phi_{0}, \mathcal{A}_{0} \in H^{1}(-\ell, \ell)$ and continuous bounded function $H(t)$ on $[0, \infty)$, Eqs.(2.1) have a unique global classical solution.

Let us first discretize the spatial interval $(-\ell, \ell)$ by $M$ equidistant gridpoints

$$
x_{i}=\ell(2 i-M-1) / M, \quad i=1, \cdots, M
$$

with spacing $h=2 \ell / M$.

For a sufficiently smooth function $w(x)$ on $[-\ell, \ell]$ with the boundary condition $D w( \pm \ell)$ $=0$, the central difference scheme leads to the following standard second order approximation

$$
\sup _{1 \leq i \leq M}\left|D^{2} w\left(x_{i}\right)-(-S \vec{w})_{i}\right| \leq C h^{2}\left|D^{4} w\right|_{0}
$$

where

$$
S=\frac{1}{h^{2}}\left(\begin{array}{rrrrrrr}
1 & -1 & 0 & \ldots & \ldots & \ldots & 0 \\
-1 & 2 & -1 & \ldots & \ldots & \ldots & 0 \\
0 & -1 & 2 & -1 & \ldots & \ldots & 0 \\
0 & \ldots & \ldots & \ldots & \ldots & \ldots & 0 \\
0 & \ldots & \ldots & -1 & 2 & -1 & 0 \\
0 & \ldots & \ldots & \ldots & -1 & 2 & -1 \\
0 & \ldots & \ldots & \ldots & 0 & -1 & 1
\end{array}\right)
$$

is an $M \times M$ matrix, $\vec{w}=\left(w\left(x_{1}\right), \cdots, w\left(x_{M}\right)\right)^{\perp}$, and $C>0$ is a generic constant.

With the notation $\vec{\psi}=\left(\psi_{1}, \cdots, \psi_{M}\right)^{\perp}, \vec{\alpha}=\left(\alpha_{1}, \cdots, \alpha_{M}\right)^{\perp}, \vec{\psi}_{0}=\left(\phi_{0}\left(x_{1}\right), \cdots\right.$, $\left.\phi_{0}\left(x_{M}\right)\right)^{\perp}$, and $\vec{\alpha}_{0}=\left(\mathcal{A}_{0}\left(x_{1}\right), \cdots, \mathcal{A}_{0}\left(x_{M}\right)\right)^{\perp}$, we may discretize (2.1) into a system of ordinary differential equations in the form

$$
\left\{\begin{array}{l}
\frac{d}{d t} \vec{\psi}=-S \vec{\psi}-P_{\psi, \alpha} \vec{\psi} \\
\frac{d}{d t} \vec{\alpha}=-S \vec{\alpha}-Q_{\psi} \vec{\alpha}-\vec{R}_{\psi}, \quad t>0 \\
\vec{\psi}=\vec{\psi}_{0}, \quad \vec{\alpha}=\vec{\alpha}_{0}, \quad t=0
\end{array}\right.
$$

where

$$
\begin{gathered}
P_{\psi, \alpha}=\lambda \operatorname{diag}\left\{\left(\psi_{1}^{2}-1\right)+H^{2}(t)\left(\alpha_{1}+x_{1}\right)^{2}, \cdots,\left(\psi_{N}^{2}-1\right)+H^{2}(t)\left(\alpha_{M}+x_{M}\right)^{2}\right\} \\
Q_{\psi}=\operatorname{diag}\left\{\psi_{1}^{2}, \cdots, \psi_{M}^{2}\right\}
\end{gathered}
$$

are diagonal matrices and $\vec{R}_{\psi}=\left(\psi_{1}^{2} x_{1}, \cdots, \psi_{M}^{2} x_{M}\right)^{\perp}$. The system (2.3) can then be integrated numerically by standard methods.

Our convergence result for the above semi-discretized problem is stated as follows. 
Theorem 2.1. Suppose $\phi_{0}, \mathcal{A}_{0} \in H^{5}(-\ell, \ell)$ and $(\phi, \mathcal{A})(x, t)$ and $(\vec{\psi}, \vec{\alpha})(t)$ are the unique global solutions of Eqs.(2.1) and (2.3), respectively. Then there exist constants $\delta, \epsilon>0$ depending only on $\ell, \lambda,\left\|\phi_{0}\right\|_{5},\left\|\mathcal{A}_{0}\right\|_{5}$, and $\sup _{t \geq 0}|H(t)|$ such that for any $T>0$, we have the pointwise error estimate

$$
\sup _{1 \leq i \leq M, 0 \leq t \leq T} \max \left\{\left|\phi\left(x_{i}, t\right)-\psi_{i}(t)\right|,\left|\mathcal{A}\left(x_{i}, t\right)-\alpha_{i}(t)\right|\right\} \leq \delta h^{2} T e^{\epsilon T} .
$$

The inequality (2.4) will be established in Section 3. In the rest part of this section, we only show that the initial value problem (2.3) has a (unique) global solution for any $M>0$.

Lemma 2.2. Set $K=\max \left\{1,\left|\phi_{0}\right|_{0}\right\}$ and $K^{\prime}=\max \left\{\ell,\left|\mathcal{A}_{0}\right|_{0}\right\}$. For a (local) solution $(\vec{\psi}, \vec{\alpha})(t)$ of Eqs.(2.3), there hold the following bounds

$$
\sup _{t \geq 0} \max _{i}\left\{\left|\psi_{i}(t)\right|\right\} \leq K, \quad \sup _{t \geq 0} \max _{i}\left\{\left|\alpha_{i}(t)\right|\right\} \leq K^{\prime} .
$$

Proof. Assume there is a $T>0$ such that $\max _{i}\left|\psi_{i}(T)\right|>K$. Choose $i_{0}: 1 \leq i_{0} \leq M$ and $t_{0} \in(0, T]$ to achieve

$$
\pm \psi_{i_{0}}\left(t_{0}\right)=\sup _{0 \leq t \leq T} \max _{i}\left\{\left|\psi_{i}(t)\right|\right\}
$$

Suppose first $\psi_{i_{0}}\left(t_{0}\right)>0$. Letting $i=i_{0}$ and $t=t_{0}$ in $(2.3 \mathrm{a})$, we have $-(S \vec{\psi})_{i_{0}} \leq 0$ and $d \psi_{i_{0}} / d t \geq 0$. So $\left(P_{\psi, \alpha} \vec{\psi}\left(t_{0}\right)\right)_{i_{0}} \leq 0$. This is false. Similarly one verifies $\psi_{i_{0}}\left(t_{0}\right) \nless 0$. This contradiction implies the validity of the bound for $\left|\psi_{i}(t)\right|$.

Let us now prove the bound for $\left|\alpha_{i}(t)\right|$. In fact, from (2.3b), we have

$$
\frac{d}{d t} \beta_{i}^{+} \geq-\left(S \vec{\beta}^{+}\right)_{i}-\psi_{i}^{2} \beta_{i}^{+}
$$

where $\beta_{i}^{ \pm}=\alpha_{i} \pm K^{\prime}$ and $\vec{\beta}^{ \pm}=\left(\beta_{i}^{ \pm}, \cdots, \beta_{M}^{ \pm}\right)^{\perp}$.

Obviously, $\beta_{i}^{+} \geq 0$ at $t=0$. We claim: $\beta_{i}^{+} \geq 0, i=1, \cdots, M$.

Define $\gamma_{i}=\beta_{i}^{+} e^{\epsilon t}, \epsilon>0$. Then $\vec{\gamma}$ satisfies

$$
\frac{d}{d t} \gamma_{i} \geq-(S \vec{\gamma})_{i}-\left(\psi_{i}^{2}+\epsilon\right) \gamma_{i}, \quad t \geq 0
$$

It can be seen that $\gamma_{i}(t) \geq 0, i=1, \cdots, M, t \geq 0$. Otherwise, suppose there is some $T>0$ which makes $\min _{1 \leq i \leq M}\left\{\gamma_{i}(T)\right\}<0$. Therefore we can choose $i_{0}: 1 \leq i_{0} \leq M$ and $t_{0} \in(0, T]$ such that

$$
\gamma_{i_{0}}\left(t_{0}\right)=\min _{0 \leq t \leq T} \min _{0 \leq i \leq M}\left\{\gamma_{i}(t)\right\} .
$$

It is easily verified that, at $t=t_{0},-(S \vec{\gamma})_{i_{0}} \geq 0$ and $d \gamma_{i_{0}} / d t \leq 0$, which yields $\gamma_{i_{0}}\left(t_{0}\right) \geq 0$ since $\gamma_{i_{0}}$ satisfies (2.5) and $\epsilon>0$. This is a contradiction. Thus the conclusion $\beta_{i}^{+} \geq 0, i=$ $1, \cdots, M$ follows. Similarly one establishes $\beta_{i}^{-} \leq 0, i=1, \cdots, M$.

The lemma is proved.

In particular, the global solvabilty of Eqs.(2.3) follows. 
3. Proof of convergence. In this section we obtain the error control (2.4). In $\mathbb{R}^{M}$, define the integral inner product and the associated norm by

$$
(\vec{u}, \vec{v})_{(h)}=h \sum_{i=1}^{N} u_{i} v_{i}, \quad\|\vec{u}\|_{(h)}^{2}=(\vec{u}, \vec{u})_{(h)}, \quad \vec{u}, \vec{v} \in \mathbb{R}^{M}
$$

Let $\vec{u} \in \mathbb{R}^{M}$. There holds

$$
(\vec{u}, S \vec{u})_{(h)}=h^{-1} \sum_{i=1}^{M-1}\left(u_{i}-u_{i+1}\right)^{2} .
$$

For given $\vec{u} \in \mathrm{R}^{M}$, choose $i_{0}: 1 \leq i_{0} \leq M$ be such that

$$
u_{i_{0}}^{2}=\min \left\{u_{1}^{2}, \cdots, u_{M}^{2}\right\} .
$$

Then, by virtue of (3.1), we have

$$
\begin{gathered}
u_{i}^{2} \leq u_{i_{0}}^{2}+\left(\sum_{j=1}^{M-1}\left(u_{j}+u_{j+1}\right)^{2} h\right)^{\frac{1}{2}}\left(\sum_{j=1}^{M-1}\left(u_{j}-u_{j+1}\right)^{2} h^{-1}\right)^{\frac{1}{2}} \\
\leq\|\vec{u}\|_{(h)}^{2} / 2 \ell+2\|\vec{u}\|_{(h)}(\vec{u}, S \vec{u})_{(h)}^{\frac{1}{2}} .
\end{gathered}
$$

This simple inequality will be used in establishing the pointwise error estimate (2.4).

Lemma 3.1. Suppose $\phi_{0}, \mathcal{A}_{0} \in H^{k}(-\ell, \ell)(k \geq 1)$ and $(\phi, \mathcal{A})(t)$ is the unique classical solution of Eqs.(2.1). There exists a constant $C_{1}$ depending only on $\ell, \lambda, \sup _{t}|H(t)|,\left\|\phi_{0}\right\|_{k}$, and $\left\|\mathcal{A}_{0}\right\|_{k}$ such that

$$
\sup _{t \geq 0} \max \left\{\|\phi(t)\|_{k},\|\mathcal{A}(t)\|_{k}\right\} \leq C_{1}
$$

Proof. The case $k=1$ has been proved in [20]. The general case is easily verified by induction.

Under the condition of Theorem 2.1, we put

$$
\vec{\phi}(t)=\left(\phi\left(x_{1}, t\right), \cdots, \phi\left(x_{M}, t\right)\right)^{\perp}, \quad \overrightarrow{\mathcal{A}}(t)=\left(\mathcal{A}\left(x_{1}, t\right), \cdots, \mathcal{A}\left(x_{M}, t\right)\right)^{\perp} .
$$

Then, by virtue of (2.2), we can rewrite Eqs.(2.1) in the form

$$
\left\{\begin{array}{l}
\frac{d}{d t} \vec{\phi}=-S \vec{\phi}-P_{\phi, \mathcal{A}} \vec{\phi}+\vec{\eta}_{1}, \\
\frac{d}{d t} \overrightarrow{\mathcal{A}}=-S \overrightarrow{\mathcal{A}}-Q_{\phi} \overrightarrow{\mathcal{A}}-\vec{R}_{\phi}+\vec{\eta}_{2}, \quad t>0 ; \\
\vec{\phi}=\vec{\psi}_{0}, \quad \overrightarrow{\mathcal{A}}=\vec{\alpha}_{0}, \quad t=0,
\end{array}\right.
$$


where $P_{\phi, \mathcal{A}}, Q_{\phi}$, and $\vec{R}_{\phi}$ are as defined in Section 2 and $\vec{\eta}_{1}(t), \vec{\eta}_{2}(t)$ satisfy

$$
\left\|\vec{\eta}_{1}(t)\right\|_{(h)}, \quad\left\|\vec{\eta}_{2}(t)\right\|_{(h)} \leq C h^{2}
$$

with $C>0$ depending only on $\ell, \lambda,\left\|\phi_{0}\right\|_{5}$, and $\left\|\mathcal{A}_{0}\right\|_{5}$ as can be seen from Lemma 3.1.

From Eqs.(2.3) and (3.3) one obtains the governing equations for the errors $\vec{\xi}=\vec{\phi}-\vec{\psi}$ and $\vec{\zeta}=\overrightarrow{\mathcal{A}}-\vec{\alpha}$ as follows:

$$
\left\{\begin{array}{l}
\frac{d}{d t} \vec{\xi}=-S \vec{\xi}-\left(P_{\phi, \mathcal{A}} \vec{\phi}-P_{\psi, \alpha} \vec{\psi}\right)+\vec{\eta}_{1}, \\
\frac{d}{d t} \vec{\zeta}=-S \vec{\zeta}-\left(Q_{\phi} \overrightarrow{\mathcal{A}}-Q_{\psi} \vec{\alpha}\right)-\left(\vec{R}_{\phi}-\vec{R}_{\psi}\right)+\vec{\eta}_{2}, \quad t>0 \\
\vec{\xi}=0, \quad \vec{\zeta}=0, \quad t=0
\end{array}\right.
$$

On the other hand, Lemmas 2.2 can be used to establish the estimates

$$
\begin{gathered}
\left\|P_{\phi, \mathcal{A}} \vec{\phi}-P_{\psi, \alpha} \vec{\psi}\right\|_{(h)} \leq C_{1}\left(\|\vec{\xi}\|_{(h)}+\|\vec{\zeta}\|_{(h)}\right) \\
\left\|Q_{\phi} \overrightarrow{\mathcal{A}}-Q_{\psi} \vec{\alpha}\right\|_{(h)} \leq C_{1}\left(\|\vec{\xi}\|_{(h)}+\|\vec{\zeta}\|_{(h)}\right) \\
\left\|\vec{R}_{\phi}-\vec{R}_{\psi}\right\|_{(h)} \leq C_{1}\|\vec{\xi}\|_{(h)},
\end{gathered}
$$

with $C_{1}$ depending only on $K, K^{\prime}$, and $\sup _{t \geq 0}|H(t)|$.

Using (3.4)-(3.6), the fact that $S \geq 0$, and the Gronwall inequality, we immediately reach the following error control in the integral norm:

$$
\|\vec{\xi}(t)\|_{(h)}^{2}+\|\vec{\zeta}(t)\|_{(h)}^{2} \leq C_{2} T h^{4} e^{C_{4} T}, \quad 0 \leq t \leq T
$$

where $C_{2}, C_{3}>0$ depend on $C, C_{1}$.

To get the expected pointwise error estimate, we need to find suitable bounds for $(\vec{\xi}, S \vec{\xi})_{(h)}$ and $(\vec{\zeta}, S \vec{\zeta})_{(h)}$.

From (3.4)-(3.7) and a simple interpolation inequality, it follows that

$$
\begin{gathered}
\frac{1}{2} \frac{d}{d t}\left((\vec{\xi}, S \vec{\xi})_{(h)}+(\vec{\zeta}, S \vec{\zeta})_{(h)}\right) \\
\leq-\|S \vec{\xi}\|_{(h)}^{2}-\|S \vec{\zeta}\|_{(h)}^{2}+\left(\left\|P_{\phi, \mathcal{A}} \vec{\phi}-P_{\psi, \alpha} \vec{\psi}\right\|_{(h)}+\left\|\vec{\eta}_{1}\right\|_{(h)}\right)\|S \vec{\xi}\|_{(h)} \\
+\left(\left\|Q_{\phi} \overrightarrow{\mathcal{A}}-Q_{\psi} \vec{\alpha}\right\|_{(h)}+\left\|\vec{R}_{\phi}-\vec{R}_{\psi}\right\|_{(h)}+\left\|\vec{\eta}_{2}\right\|_{(h)}\right)\|S \vec{\zeta}\|_{(h)}
\end{gathered}
$$




$$
\leq C_{4} h^{4} e^{C_{5} T}, \quad 0 \leq t \leq T
$$

with $C_{4}, C_{5}>0$ depending only on $C, C_{2}, C_{3}$, which implies the bound

$$
(\vec{\xi}, S \vec{\xi})_{(h)}(t)+(\vec{\zeta}, S \vec{\zeta})_{(h)}(t) \leq T C_{4} h^{4} e^{C_{5} T}, \quad 0 \leq t \leq T
$$

Finally, combining (3.2), (3.7), and (3.8), one achieves the following pointwise error control

$$
\sup _{1 \leq i \leq M, 0 \leq t \leq T} \max \left\{\left|\xi_{i}(t)\right|,\left|\zeta_{i}(t)\right|\right\} \leq T C_{6} h^{2} e^{C_{7} T}
$$

where $C_{6}, C_{7}>0$ are determined by $\ell$ and $C_{j}(j=2, \cdots, 5)$.

The proof of Theorem 2.1 is complete.

Now the semi-discretized system (2.3) may further be discretized in the time variable by various finite difference schemes. For our purpose, we choose to use the backward or implicit difference scheme to discretize the time. In this manner, although (2.3) is nonlinear, a maximum principle argument enables us to prove the stability of the scheme in the pointwise norm and, then, the convergence will follow readily. Such an analysis is presented in the next section. A series of numerical results obtained by this scheme and their interesting physical implications will be detailed in Section 5 .

4. Analysis of the backward difference scheme. On a given finite time interval $[0, T], T>0$, let $k=T / N$ (where $N>0$ is an integer) be the discretized time step and

$$
t_{j}=j k, \quad j=0,1, \cdots, N
$$

the mesh points. With the notation

$$
\begin{gathered}
\phi_{i}^{j}=\phi\left(x_{i}, t_{j}\right), \quad \mathcal{A}_{i}^{j}=\mathcal{A}\left(x_{i}, t_{j}\right), \quad i=1, \cdots, M, \quad j=0,1, \cdots, N, \\
\triangle^{2} u_{i}=u_{i+1}-2 u_{i}+u_{i-1}, \quad i=1, \cdots, M,
\end{gathered}
$$

and after replacing $\phi_{t}$ and $\mathcal{A}_{t}$ at $x=x_{i}, t=t_{j+1}$ by $\left(\phi_{i}^{j+1}-\phi_{i}^{j}\right) / k$ and $\left(\mathcal{A}_{i}^{j+1}-\mathcal{A}_{i}^{j}\right) / k$, we can rewrite $(2.1)$ as

$$
\left\{\begin{array}{l}
\phi_{i}^{j+1}=\phi_{i}^{j}+r \triangle^{2} \phi_{i}^{j+1}-\lambda k\left(\left[\phi_{i}^{j+1}\right]^{2}-1+H^{2}\left(t_{j+1}\right)\left[\mathcal{A}_{i}^{j+1}+x_{i}\right]^{2}\right) \phi_{i}^{j+1}+\mu_{i}^{j+1} \\
\mathcal{A}_{i}^{j+1}=\mathcal{A}_{i}^{j}+r \triangle^{2} \mathcal{A}_{i}^{j+1}-k\left(\phi_{i}^{j+1}\right)^{2}\left(\mathcal{A}_{i}^{j+1}+x_{i}\right)+\nu_{i}^{j+1}, \quad j=0,1, \cdots, N-1 \\
\phi_{i}^{0}=\phi_{0}\left(x_{i}\right), \quad \mathcal{A}_{i}^{0}=\mathcal{A}_{0}\left(x_{i}\right)
\end{array}\right.
$$

where $r=k / h^{2}, \phi_{0}^{j}=\phi_{1}^{j}, \phi_{M}^{j}=\phi_{M+1}^{j}, \mathcal{A}_{0}^{j}=\mathcal{A}_{1}^{j}, \mathcal{A}_{M}^{j}=\mathcal{A}_{M+1}^{j}, i=1, \cdots, M$, and $\mu_{i}^{j}, \nu_{i}^{j}$ satisfy

$$
k E_{T} \equiv \max _{i, j}\left\{\left|\mu_{i}^{j}\right|,\left|\nu_{i}^{j}\right|\right\} \leq C\left(k^{2}+k h^{2}\right) \sup _{-\ell \leq x \leq \ell, 0 \leq t \leq T}\left\{\left|\phi_{t t}\right|,\left|\mathcal{A}_{t t}\right|,\left|D^{4} \phi\right|,\left|D^{4} \mathcal{A}\right|\right\} .
$$


Therefore, neglecting the error terms $\mu_{i}^{j}, \nu_{i}^{j}$, and replacing $\phi_{i}^{j}, \mathcal{A}_{i}^{j}$ by $\psi_{i}^{j}, \alpha_{i}^{j}$ in (4.1), we have the following implicit scheme

$$
\left\{\begin{array}{l}
\psi_{i}^{j+1}=\psi_{i}^{j}+r \triangle^{2} \psi_{i}^{j+1}-\lambda k\left(\left[\psi_{i}^{j+1}\right]^{2}-1+H^{2}\left(t_{j+1}\right)\left[\alpha_{i}^{j+1}+x_{i}\right]^{2}\right) \psi_{i}^{j+1} \\
\alpha_{i}^{j+1}=\alpha_{i}^{j}+r \triangle^{2} \alpha_{i}^{j+1}-k\left(\psi_{i}^{j+1}\right)^{2}\left(\alpha_{i}^{j+1}+x_{i}\right), \quad j=0,1, \cdots, N-1 \\
\psi_{i}^{0}=\phi_{0}\left(x_{i}\right), \quad \alpha_{i}^{0}=\mathcal{A}_{0}\left(x_{i}\right),
\end{array}\right.
$$

with the boundary condition $\psi_{0}^{j}=\psi_{1}^{j}, \psi_{M}^{j}=\psi_{M+1}^{j}, \alpha_{0}^{j}=\alpha_{1}^{j}, \alpha_{M}^{j}=\alpha_{M+1}^{j}$.

The following lemma concerning the stability of the backward scheme (4.2) is easily proved by a maximum principle argument.

Lemma 4.1. With the notation $K=\max \left\{1,\left|\phi_{0}\right|_{0}\right\}$ and $K^{\prime}=\max \left\{\ell,\left|\mathcal{A}_{0}\right|_{0}\right\}$ (see Lemma 2.2), we have $\left|\psi_{i}^{j}\right| \leq K$ and $\left|\alpha_{i}^{j}\right| \leq K^{\prime}$.

Proof. Suppose otherwise there were $i_{0}: 1 \leq i_{0} \leq M$ and $j_{0}: 1 \leq j_{0} \leq N$ such that

$$
\psi_{i_{0}}^{j_{0}}=\sup _{i, j} \psi_{i}^{j}>K
$$

Then the structure of Eq.(4.2) would lead to a contradiction under the substitution $j+1=$ $j_{0}, i=i_{0}$. Hence $\psi_{i}^{j} \leq K$. Similarly one shows $\psi_{i}^{j} \geq-K$.

We now verify the bound for $\left|\alpha_{i}^{j}\right|$. Suppose $\sup \alpha_{i}^{j}>K^{\prime}$. Let $j_{0}: 1 \leq j_{0} \leq N$ satisfy

$$
j_{0}=\min \left\{j^{\prime}=1, \cdots, N \mid \alpha_{i_{0}}^{j^{\prime}}=\max _{i, j} \alpha_{i}^{j}\right\}
$$

Inserting $j_{0}=j+1, i=i_{0}$ into $(4.2)$ we find $\left(\psi_{i_{0}}^{j_{0}}\right)^{2}\left(\alpha_{i_{0}}^{j_{0}}+x_{i_{0}}\right)<0$. This violates our assumption. By the same way, we can prove $\inf \alpha_{i}^{j} \geq-K^{\prime}$. So the lemma follows.

From Eqs.(4.1) and (4.2), the governing equations for the errors $\xi_{i}^{j}=\phi_{i}^{j}-\psi_{i}^{j}, \zeta_{i}^{j}=$ $\mathcal{A}_{i}^{j}-\alpha_{i}^{j}$ are:

$$
\left\{\begin{array}{l}
r \triangle^{2} \xi_{i}^{j+1}-\xi_{i}^{j+1}=f_{i}^{j+1} \\
r \triangle^{2} \zeta_{i}^{j+1}-\zeta_{i}^{j+1}=g_{i}^{j+1} \\
\xi_{i}^{0}=0, \quad \zeta_{i}^{0}=0
\end{array}\right.
$$

where

$$
\begin{aligned}
f_{i}^{j+1}= & -\xi_{i}^{j}+\lambda k\left(\left[\phi_{i}^{j+1}\right]^{2}-1+H^{2}\left(t_{j+1}\right)\left[\mathcal{A}_{i}^{j+1}+x_{i}\right]^{2}\right) \phi_{i}^{j+1} \\
& -\lambda k\left(\left[\psi_{i}^{j+1}\right]^{2}-1+H^{2}\left(t_{j+1}\right)\left[\alpha_{i}^{j+1}+x_{i}\right]^{2}\right) \psi_{i}^{j+1}-\mu_{i}^{j+1}, \\
g_{i}^{j+1}= & -\zeta_{i}^{j}+k\left(\phi_{i}^{j}\right)^{2}\left(\mathcal{A}_{i}^{j+1}+x_{i}\right)-k\left(\psi_{i}^{j}\right)^{2}\left(\alpha_{i}^{j+1}+x_{i}\right)-\nu_{i}^{j+1} .
\end{aligned}
$$


Using the boundary condition $\xi_{0}^{j}=\xi_{1}^{j}, \xi_{M}^{j}=\xi_{M+1}^{j}, \zeta_{0}^{j}=\zeta_{1}^{j}, \zeta_{M}^{j}=\zeta_{M+1}^{j}$, and a maximum principle argument in (4.3) as in [8], we find

$$
\max _{i}\left|\xi_{i}^{j+1}\right| \leq \max _{i}\left|f_{i}^{j+1}\right|, \quad \max _{i}\left|\zeta_{i}^{j+1}\right| \leq \max _{i}\left|g_{i}^{j+1}\right|
$$

Therefore, it follows from Lemma 4.1 and (4.4) that

$$
\begin{aligned}
\max _{i}\left|\xi_{i}^{j+1}\right| \leq \max _{i}\left|\xi_{i}^{j}\right|+\lambda k\left(3 K^{2}+\sup _{t} H^{2}(t)\left(K^{\prime 2}+2 \ell K^{\prime}+\ell^{2}\right)+1\right) \max _{i}\left|\xi_{i}^{j+1}\right| \\
+2 \lambda k K \sup _{t} H^{2}(t)\left(K^{\prime}+\ell\right) \max _{i}\left|\zeta_{i}^{j+1}\right|+\max _{i}\left|\mu_{i}^{j+1}\right|, \\
\max _{i}\left|\zeta_{i}^{j+1}\right| \leq \max _{i}\left|\zeta_{i}^{j}\right|+2 k K\left(K^{\prime}+\ell\right) \max _{i}\left|\xi_{i}^{j+1}\right|+k K^{2} \max _{i}\left|\zeta_{i}^{j+1}\right|+\max _{i}\left|\nu_{i}^{j+1}\right| .
\end{aligned}
$$

Define

$$
\begin{aligned}
\theta= & \max \left\{\lambda\left(3 K+\sup _{t} H^{2}(t)\left(K^{\prime 2}+2 \ell K^{\prime}+\ell^{2}\right)+1\right)\right. \\
& \left.+2 K\left(K^{\prime}+\ell\right), 2 \lambda K \sup _{\boldsymbol{t}} H^{2}(t)\left(K^{\prime}+\ell\right)+K^{2}\right\} .
\end{aligned}
$$

Given $\epsilon \in(0,1)$, let $k$ be sufficiently small so that $k \theta \leq 1-\epsilon$. Consequently, the initial condition $\xi_{i}^{0}=0, \zeta_{i}^{0}=0$ implies the inequality

$$
\begin{gathered}
\max _{i}\left|\xi^{j+1}\right|+\max _{i}\left|\zeta_{i}^{j+1}\right| \leq(1-k \theta)^{-1}\left(\max _{i}\left|\xi_{i}^{j}\right|+\max _{i}\left|\zeta_{i}^{j}\right|\right)+2 k(1-k \theta)^{-1} E_{T} \\
\leq 2 E_{T} T(1-\theta T / N)^{-N} \leq 2 E_{T} T \epsilon^{-\theta T /(1-\epsilon)}
\end{gathered}
$$

It may be verified by Lemma 3.1, Eqs.(2.1), and the embedding

$$
H^{k}(-\ell, \ell) \rightarrow C^{k-1}[-\ell, \ell]
$$

that the conditions $\phi_{0}, \mathcal{A}_{0} \in H^{5}(-\ell, \ell)$ and $\sup _{t}(|H(t)|+|\dot{H}(t)|)<\infty$ ensure that the quantity

$$
\sup _{-\ell \leq x \leq \ell, t \geq 0} \max \left\{\left|\phi_{t t}(x, t)\right|,\left|\mathcal{A}_{t t}(x, t)\right|,\left|D^{4} \phi(x, t)\right|,\left|D^{4} \mathcal{A}(x, t)\right|\right\}
$$

can be bound by a constant depending only on $\ell, \lambda,\left\|\phi_{0}\right\|_{5},\left\|\mathcal{A}_{0}\right\|_{5}$, and $\sup _{t}(|H(t)|+|\dot{H}(t)|)$. Hence, we have proved 
Theorem 4.2. Suppose $(\phi(x, t), \mathcal{A}(x, t))$ is the solution of Eqs.(2.1) where $\phi_{0}, \mathcal{A}_{0} \in$ $H^{5}(-\ell, \ell)$ and $H(t)$ is a $C^{1}$ function so that $\sup _{t \geq 0}(|H(t)|+|\dot{H}(t)|)<\infty$ and $\left\{\left(\psi_{i}^{j}, \alpha_{i}^{j}\right)\right\}$ the sequence determined by the backward scheme (4.2). Let $\theta$ be as defined in (4.5). For any $\epsilon \in(0,1)$, we have the error estimate

$$
\max \left\{\left|\phi\left(x_{i}, t_{j}\right)-\psi_{i}^{j}\right|,\left|\mathcal{A}\left(x_{i}, t_{j}\right)-\alpha_{i}^{j}\right|\right\} \leq \delta\left(k+h^{2}\right) T \epsilon^{-\theta T /(1-\epsilon)}
$$

where $k \theta \leq 1-\epsilon$ or $N \geq \theta T /(1-\epsilon)$ and $\delta>0$ is a constant depending only on $\ell, \lambda,\left\|\phi_{0}\right\|_{5},\left\|\mathcal{A}_{0}\right\|_{5}$, and $\sup _{t \geq 0}(|H(t)|+|\dot{H}(t)|)$.

NotE. Since our purpose is to calculate the physical states of a superconducting film under the influence of an external source $H=H_{0} \neq 0$ by connecting these states with the states at $H=0$, so $\phi_{0}$ and $\mathcal{A}_{0}$ are determined by (1.2):

$$
\phi_{0}= \pm 1, \quad \mathcal{A}_{0}=\sinh x / \cosh \ell-x .
$$

Therefore $K=1, K^{\prime}=\max \{1, \ell\}$, and constant $\theta$ is easily evaluated.

5. Numerical results. In this section we present a series of numerical results obtained by the gradient flow method analyzed in the previous sections. Although, in principle, the physical solutions of Eqs.(1.1) are to be found in the $t \rightarrow \infty$ limit of the solutions of Eqs.(1.4) where $\phi_{0}, A_{0}$ satisfy (1.2), our computations carried out up to a large value of the coupling constant $\lambda=1000$ show that the actual convergence time is quite short. In all of the examples here, the approximations are already impressively satisfactory after a run with $T=5-164$. This suggests that our method may provide an efficient and practical tool for computing various aspects of thin film superconductivity.

Recall that, the normal and superconducting states are characterized respectively by solutions of Eqs.(1.1) satisfying $\phi(x) \equiv 0, A^{\prime}(x) \equiv 1$ and $\phi(x) \not \equiv 0, A^{\prime}(x) \not \equiv 1$. In our experiments below we are interested in the phase transitions from normal to superconducting states, and vice versa, as one varies the external magnetic field $H$ and the coupling constant $\lambda$. A sufficient condition has been established earlier [20] which ensures the occurrence of the superconducting phase. This condition reads

$$
\ell^{2} H^{2} \leq 3 / 2
$$

Occasionally, we will use this condition as a reference for choosing the strength of the external field.

The numerical scheme used in this section is the backward finite difference method analyzed in Section 4. Let us first explain briefly the computational procedure taken here.

With the notation of Section 4, put

$$
U^{j}=\left(\psi_{1}^{j}, \cdots, \psi_{M}^{j}, \alpha_{1}^{j}, \cdots, \alpha_{M}^{j}\right), \quad j=0,1, \cdots, N .
$$


We may write Eqs.(4.2) as

$$
\mathcal{L}\left(U^{j+1}\right)=U^{j}+\Gamma\left(U^{j+1}\right)
$$

where

$$
\mathcal{L}\left(U^{j}\right)=\left(\psi_{1}^{j}-r \triangle^{2} \psi_{1}^{j}, \cdots, \psi_{M}^{j}-r \triangle^{2} \psi_{M}^{j}, \alpha_{1}^{j}-r \triangle^{2} \alpha_{1}^{j}, \cdots, \alpha_{M}^{j}-r \triangle^{2} \alpha_{M}^{j}\right),
$$

and the form of $\Gamma\left(U^{j}\right)$ is self-evident. Knowing $U^{j}$, we must determine $U^{j+1}$. Since (5.2) is an.implicit equation, an iterative method has to be introduced. The iterative sequence $\left\{U^{j+1, m}\right\}_{m=0}^{\infty}$ is defined as follows.

$$
\left\{\begin{array}{l}
U^{j+1,0}=U^{j} \\
\mathcal{L}\left(U^{j+1, m+1}\right)=U^{j}+\left(f\left(U^{j+1, m+1}, U^{j+1, m}\right), g\left(U^{j+1, m+1}, U^{j+1, m}\right)\right)
\end{array}\right.
$$

with

$$
\begin{gathered}
f\left(U^{j+1, m+1}, U^{j+1, m}\right) \\
=-\lambda k\left(H^{2}\left(t_{j+1}\right)\left[\alpha_{i}^{j+1, m}+x_{i}\right]^{2} \psi_{i}^{j+1, m+1}+\left[\left(\psi_{i}^{j+1, m}\right)^{2}-1\right] \psi_{i}^{j+1, m}\right)_{i=1}^{M} \\
g\left(U^{j+1, m+1}, U^{j+1, m}\right)=-k\left(\left[\psi_{i}^{j+1, m}\right]^{2} \alpha_{i}^{j+1, m+1}+\left[\psi_{i}^{j+1, m}\right]^{2} x_{i}\right)_{i=1}^{M} .
\end{gathered}
$$

The iteration terminates when

$$
\left|U^{j+1, m+1}-U^{j+1, m}\right| \equiv \sup _{i}\left|\psi_{i}^{j+1, m+1}-\psi_{i}^{j+1, m}\right|+\sup _{i}\left|\alpha_{i}^{j+1, m+1}-\alpha_{i}^{j+1, m}\right|<10^{-7}
$$

and $\Gamma\left(U^{j+1}\right)$ is then approximated by $\left(f\left(U^{j+1, m+1}, U^{j+1, m}\right), g\left(U^{j+1, m+1}, U^{j+1, m}\right)\right)$. In all of our experiments, the accuracy (5.3) can be reached after 5-10 steps. This completes one step in the implicit iterative scheme (5.2).

For the scheme (5.2), our calculation terminates if the accuracy

$$
\left|U^{j+1}-U^{j}\right|<10^{-8}
$$

is achieved which means that a steady state solution is found: the discretized gradient flow $\left\{U^{j}\right\}$ converges to a physical state of the superconducting film. Obviously, in different parameter regions, the required time for the computation varies greatly.

For convenience, in what follows, we will fix $\ell=1$ unless otherwise stated.

EXAMPLE 5.1. We first examine that the numerical solutions of Eqs.(1.1) at $H=H_{0}$ obtained by connecting the states at $H=0$ through the gradient flow (1.3) are independent of the choice of the "connecting function" $H(t): H(0)=0, H(t)=H_{0}, t \geq$ some $t_{0}>0$. 
For definiteness, let us set $\lambda=0.3$ (type I superconductivity), $M=101$, and $r=50$ in the scheme (4.2) or (5.2). If $H_{0}=2,(5.1)$ is violated and we may expect to find a normal state solution. Two connecting functions are taken:

$$
F(t)=\min \left\{t, H_{0}\right\}, \quad G(t)=\min \left\{0.5 t+\sin 2 \pi t, H_{0}\right\} .
$$

$F(t) \equiv H_{0}$ after $t \geq 2$ and is strictly monotone in $[0,2)$ but $G(t) \equiv H_{0}$ for $t \geq 6$ and oscillates in $[0,6)$. For both cases, the numerical solutions converge to the normal state $\phi=0, A=x$ (the computation terminates at $t=164$ ).

Figure 5.1a illustrates the energy decay corresponding to different connecting functions. Initially, the two curves differ greatly, but they soon approach the lowest energy level $E=0.15$.

Figure 5.1b displays the behaviour of the excited magnetic field $B(x, t)=H(t) D A(x, t)$ with $H(t)=F(t)$. As time $t$ increases from $t=0.48$ to 164, the transition from superconducting states to the normal state develops. Eventually, the external field completely penetrates the film and superconductivity is quenched. The curves from the bottom to the top are ordered by increasing time.

ExAmPle 5.2. Let the data $\lambda, M, r$ be the same as in Example 5.1. Choose $H=$ $H_{0}=1$ and the connecting function $H(t)=F(t)$ (cf.(5.4)). From (5.1) we know that the film is in the superconducting phase. In our computation, the energy decays after a short period of time and the numerical solutions converge to a superconducting state (the program halts at $t=33.25$ ).

Figure 5.2a shows the behaviour of the order parameter $\phi$ at different time stages. The curves from the top to the bottom are ordered by increasing time $t=0.49-33.25$. At $t=0.49$, since the external field is still weak, the film is almost in the completely superconducting state and the density of the Cooper pairs stays close to its maximum value $\phi=1$. As time develops and the external field becomes strong, the superconducting electron pairs can hardly survive near the boundary of the film and they tend to gain a greater density in the middle of the sample. This is known as the nucleation of superconductivity at intermediate external fields.

In Figure 5.2b, the graphs of the excited field versus the spatial variable $x$ are ordered from the bottom to the top by increasing time $t=0.49-33.25$. At low field, namely, $t=0.49$, the external field may only achieve a partial penetration near the surfaces and it is completely screened in the middle of the film. As time develops, the external source becomes strong and the solution steadily approaches a stable state. This state represents a superconducting phase. There is a complete penetration of the external field near the surfaces and the excited field rapidly decays to its minimum at the center of the sample. The magnetic screening is partial.

EXAMPLE 5.3. Figure 5.3 is the energy curves versus the values of the external magnetic field $H$ of the computed solutions of (1.1), the normal state, and the completely 
superconducting states given by (1.2). Here $\lambda=0.3$. These curves are denoted by the letters $\mathrm{C}, \mathrm{N}$, and $\mathrm{S}$, respectively. It is seen that the energy of the least energy solutions (the C curve) of Eqs.(1.1) is below the normal state energy level $E=0.15$ (the $\mathrm{N}$ curve) if $H<1.75$. Therefore the sample is in the superconducting phase. While, for $H>1.75$, the $\mathrm{C}$ curve joins the $\mathrm{N}$ curve and the sample is in the normal phase. When $H<0.6$, the $\mathrm{C}$ curve coincides with the $\mathrm{S}$ curve and the film is in a completely superconducting state. In the range $0.6<H<1.75$, the $\mathrm{C}$ curve is strictly below the $\mathrm{N}$, and $\mathrm{S}$, curve and the sample is in a superconducting state different from those given by (1.2). The magnetic screening is partial even at the center of the film and a symmetric nucleation of superconductivity occurs. These global energy curves provide a very clear picture of phase transitions in terms of changes of the external field and completely verify the Meissner effect.

EXAMPLE 5.4. As a comparison, we remark that the energy curves versus external fields are qualitatively different for type II superconductors. Let us choose the data $\lambda=$ $10, M=101, r=50$. The computer results are summarized in Figure 5.4. As in the above example, there are two critical fields, $H_{c_{1}}=0.5$ and $H_{c_{2}}=3$. For $H<H_{c_{1}}$, the sample is in a completely superconducting state and behaves like a piece of diamagnetic material. In the range $H_{c_{1}}<H<H_{c_{2}}$, the energy curve $\mathrm{C}$ of the computed solutions bends away from the curve $\mathrm{S}$ and the Abrikosov mixed states [1] are maintained. Finally, when $H>H_{c_{2}}$, the $\mathrm{C}$ curves joins the $\mathrm{N}$ curve and the normal phase is reached. This is a characteristic phenomenon of type II superconductivity. It is seen that, in Figure 5.3 both $\mathrm{C}$ and $\mathrm{S}$ curves are convex (although there is an inflection point on the $\mathrm{C}$ curve near the $\mathrm{N}$ curve) and tend to stay close, while, in Figure 5.4, the $\mathrm{C}$ curve is concave after $H=H_{c_{1}}$ and tends to stay away from the $\mathrm{S}$ curve. In fact, Figure 5.3 indicates a small deviation of the Ginzburg-Landau theory from physically observed facts in type I superconductors but Figure 5.4 tells us exactly what really happen in type II superconductors.

EXAMPLE 5.5. We are interested also in the behaviour of solutions when the coupling constant $\lambda$ takes very large values. Results have been obtained with fixed external field $H=H_{0}=5$ and the values of $\lambda$ varying in the range 10-1000. We still choose $M=101$. Note that the expression of $\theta$ linearly depends on $\lambda$ if $\lambda$ is large and that, by Theorem 4.2, we must require $r \lambda$ small in order to achieve convergence of the backward difference scheme (4.2) or (5.2). In our computer experiments, we find that $r \lambda=500$ seems to be optimal for the problems discussed here. The calculations terminate at $t=5-10$. The curves of the order parameter $\phi$ in Figure 5.5 are ordered from the bottom to the top by increasing values of $\lambda=10,50,100,500,1000$. It is seen that, for $\lambda=10$, the superconducting phase is destroyed and the normal state dominates. The $\phi$ curve is represented by a horizontal line $\phi=0$. For larger $\lambda$, a superconducting region may be maintained near the center of the sample but finite normal conducting layers also exist. This shows the co-existence of both superconducting and normal regions in a film at intermediate fields in type II superconductivity. As $\lambda$ increases, the peak of the $\phi$ curve grows and the density of the 


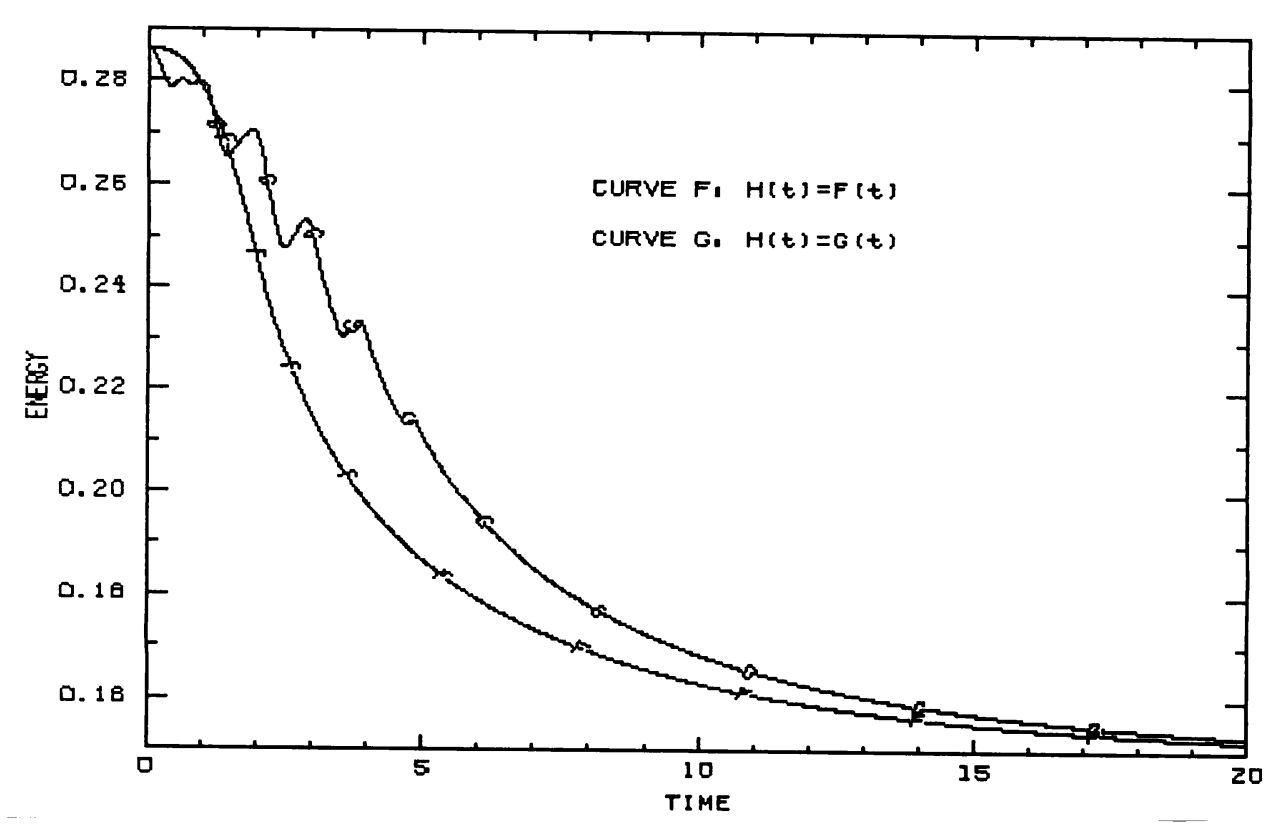

FIGURE 5.1 (a)

Energy decay curves corresponding to two different connecting functions: Although the energies differ initially, after a period of time they both reach the least energy level $E=0.15$ of the system at $H=2$ and the sample then stays in the normal phase. Thus, the evolution history is not important in the Ginzburg-Landau theory.

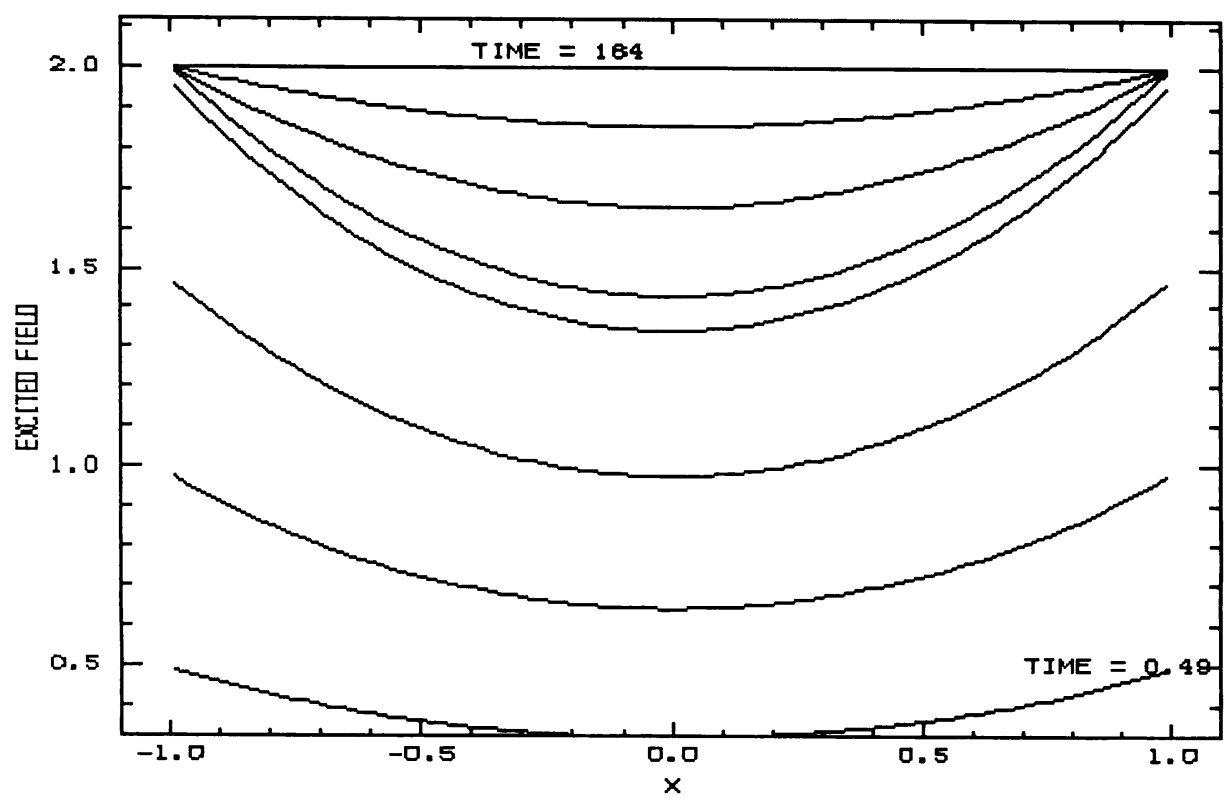

FIGURE 5.1 (b)

Development of the state from the completely superconducting phase to the normal phase: Eventually no superconducting electron pairs can survive and the sample is penetrated by the external field like a normal conductor. 


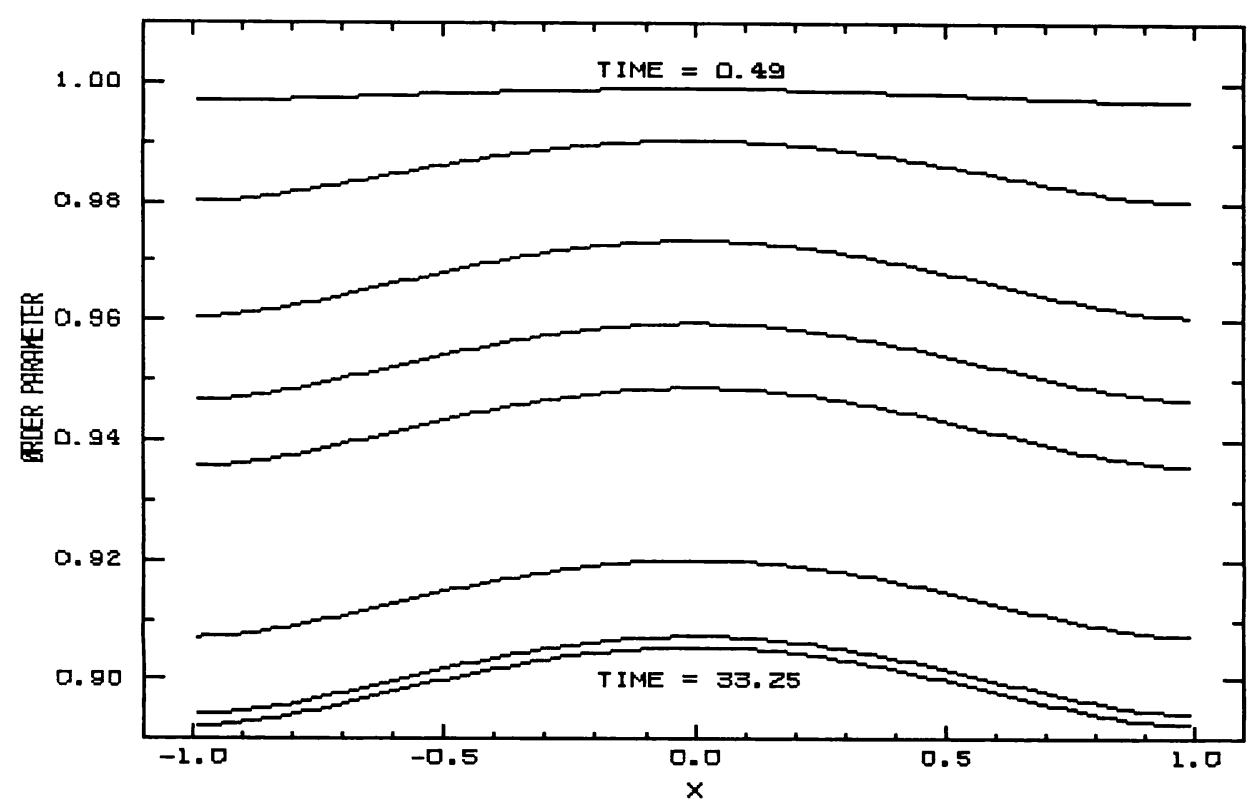

FIGURE $5.2(\mathrm{a})$

Behaviour of the order parameter $\phi$ at different time stages: In the large time limit the flow approaches a least energy solution of the system and the sample is in a superconducting state so that a symmetric nucleation of superconductivity is maintained. The strength of the external field is neither weak enough to leave the film in the completely superconducting states nor sufficiently strong to destroy superconductivity.

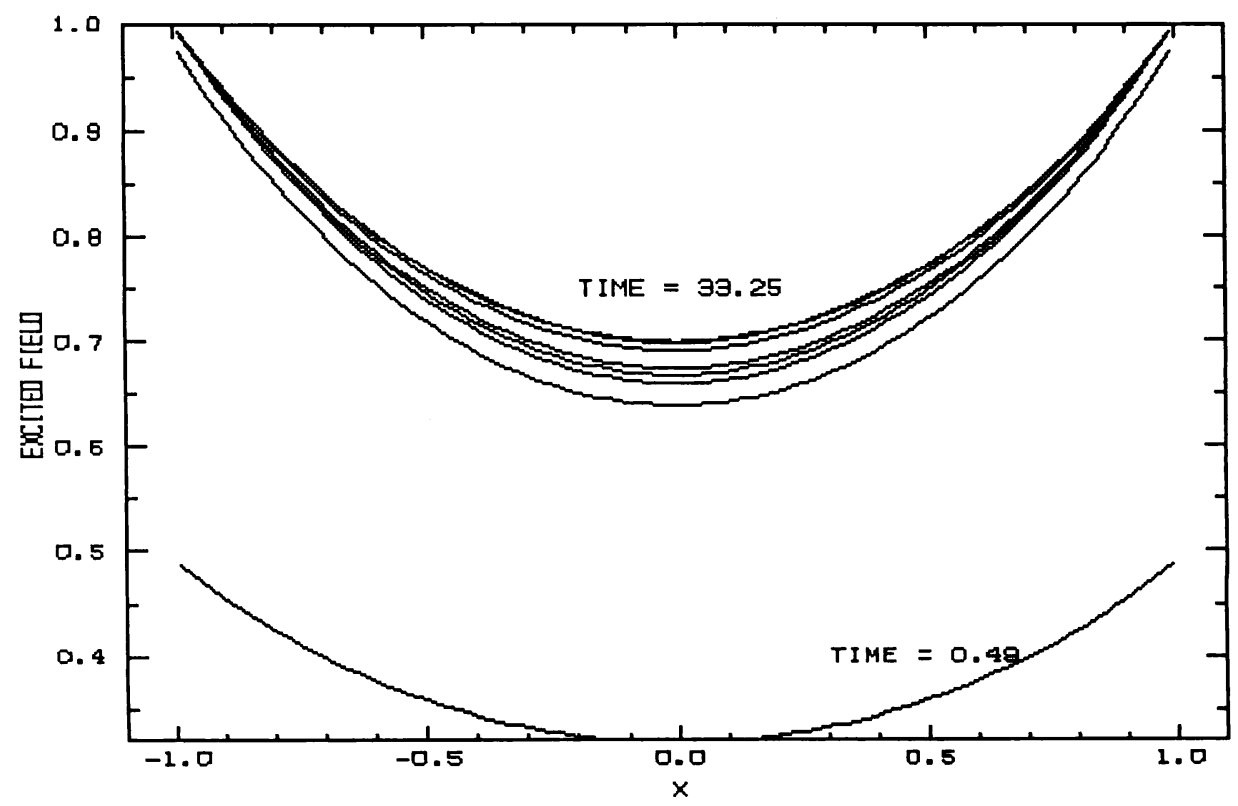

FIGURE $5.2(\mathrm{~b})$

Partial penetration of the external field: The regions near the surfaces of the film are almost in the normal state due to the influence of a sufficiently strong external source and the penetration strength attains its minimum at the center of the sample. 


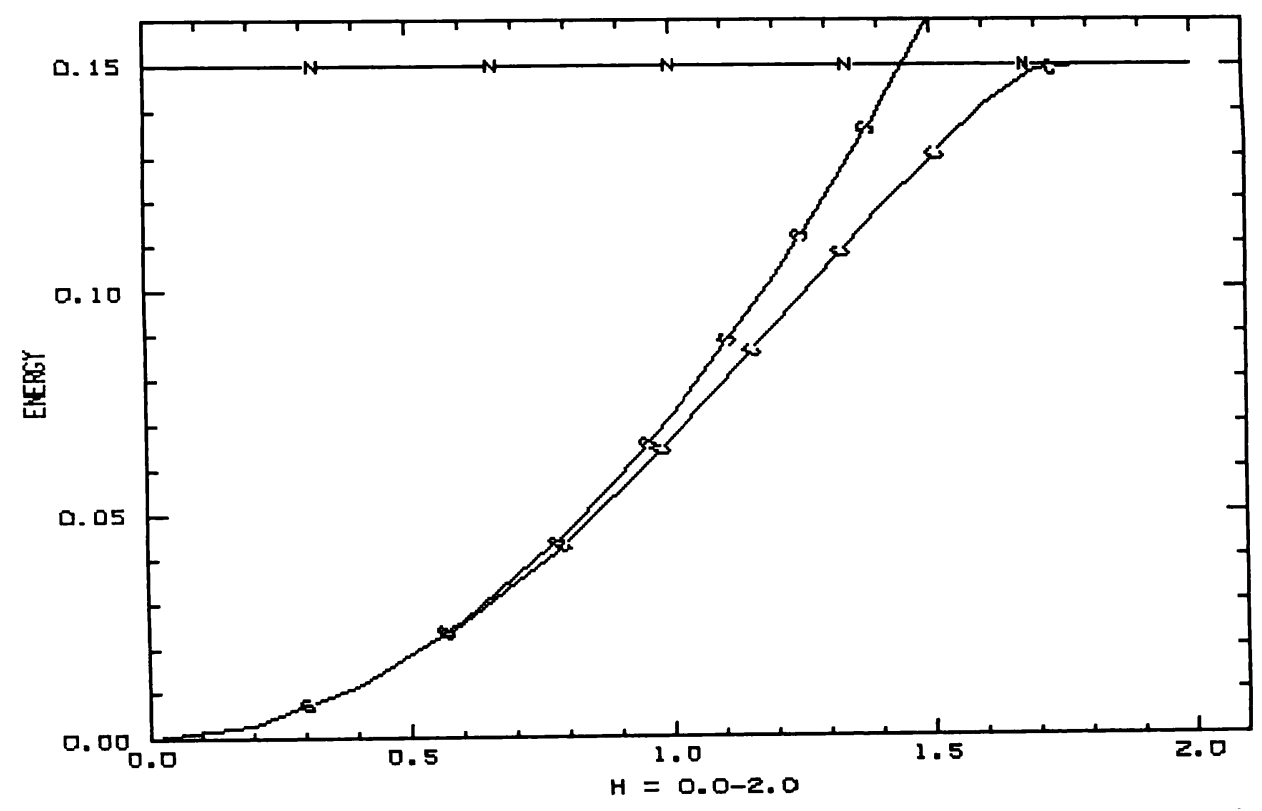

FIGURE 5.3

Three branches of energy curves versus the values of the external magnetic field: Letters $\mathrm{C}, \mathrm{N}, \mathrm{S}$ denote the energy curves of the computed solutions, the normal state, and the completely superconducting states (given by (1.2)), respectively. Physical experiments say that the $\mathrm{C}$ curve should simply coincides with the $\mathrm{S}$ curve (at low fields) and with the $\mathrm{N}$ curve (at high fields) since $\lambda=0.3$ corresponds to type I superconductivity. Our results here show that there is a slight deviation of the Ginzburg-Landau theory from experimental facts at intermediate fields.

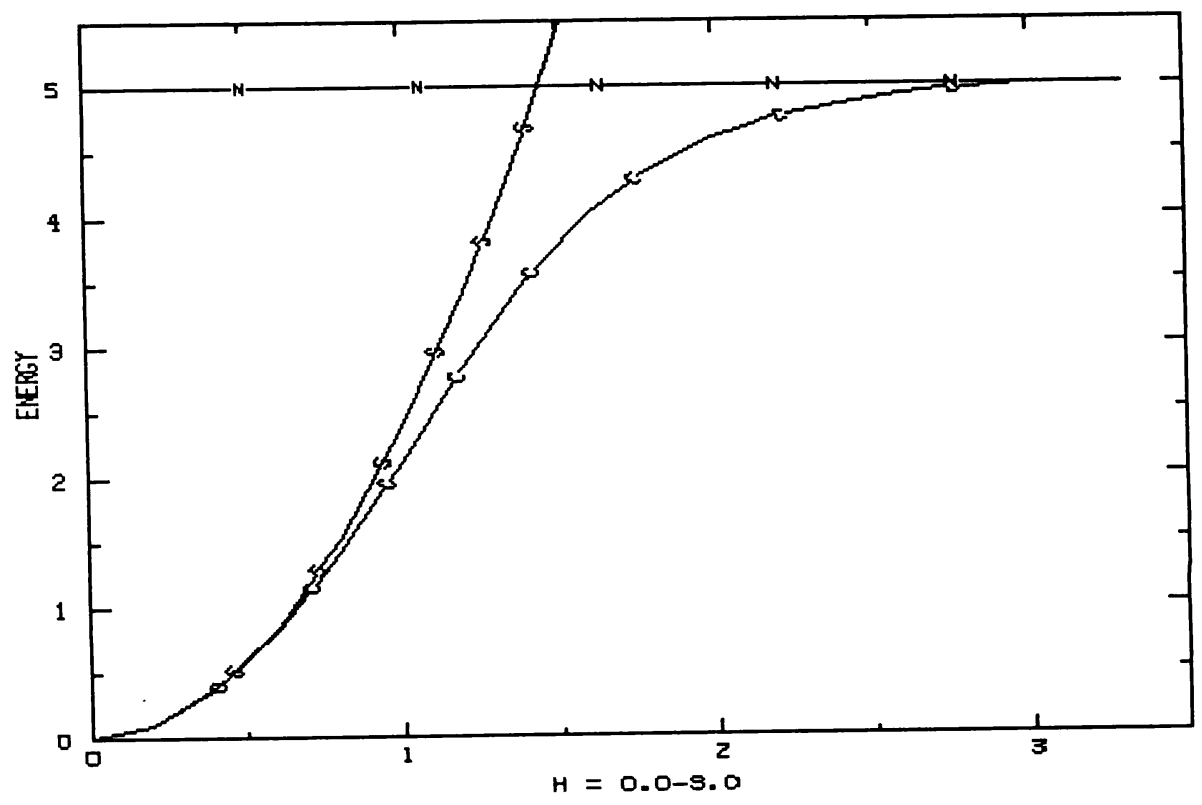

FIGURE 5.4

Energy curves versus external fields in type II superconductors: The magnetic behaviour of the sample is now characterized by two critical fields $H_{c_{1}}=0.5$ and $H_{c_{2}}=3$ which substantially differs from that in type I superconductors. When $H>H_{c_{1}}$, the realistic energy curve $\mathrm{C}$ will bend away from the $\mathrm{S}$ curve and finally join the $\mathrm{N}$ curve. 


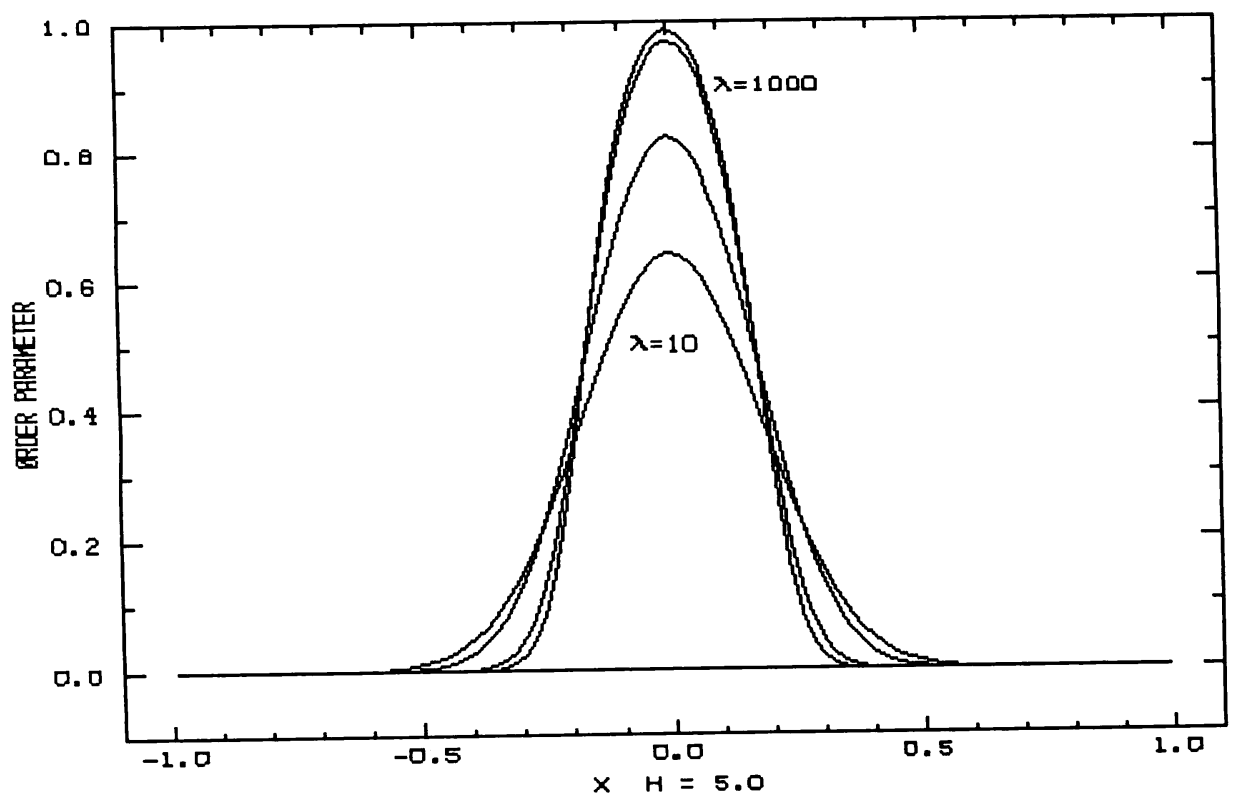

Figure 5.5

Large $\lambda$ behaviour of superconducting films: Although the field $H=5$ is strong enough to quench superconductivity in samples with $\lambda \leq 10$, large $\lambda$ materials can still maintain a superconducting state in regions near the center. In this case superconducting and normal states co-exist. As $\lambda$ grows, the superconducting core is squeezed into a very narrow region. 


\section{REFERENCES}

[1] A. A. Abrikosov, On the magnetic properties of superconductors of the second group, Sov. Phys. JETP 5(1957), 1174-1182.

[2] V. Ambegaokar, Critical currents and the onset of resistence in superconductors, in Superconductivity, edited by P. A. Wallace, Gordon and Breach, New York, 1969, pp.117-158.

[3] M. S. Berger, Y. Y. Chen, Symmetric vortices for the Ginzburg-Landau equations and the nonlinear desingularization phenomenon, J. Funct. Anal. (to appear).

[4] M. S. Berger, L. E. Fraenkel, On nonlinear desingularization, Bull. Amer. Math. Soc. 2(1980), $165-167$.

[5] J. P. Burger, Superheating and supercooling in first kind superconductors, in Superconductivity, edited by P. A. Wallace, Gordon and Breach, New York, 1969, pp.463-488.

[6] P. H. Damgand, U. M. Heller, Observation of the Meissner effect in a lattice Higgs model, Phys. Rev. Lett. 60(1988), 1246-1249.

[7] T. A. DeGrand, D. Toussaint, Topological excitations and Monte Carlo simulation of abelian gauge theory, Phys. Rev D 22(1980), 2478-2489.

[8] J. Douglas, JR., On the numerical integration of quasi-linear parabolic differential equations, Pacific J. Math. 6(1956), 35-42.

[9] J. Eells, L. Lemaire, A report on harmonic maps, Bull. London Math. Soc. 10(1978), 1-68.

[10] J. Eells, J. M. Sampson, Harmonic mappings of Riemannian manifolds, Amer. J. Math. 86(1964), $109-160$.

[11] V. L. Ginzburg, L. D. Landau, On the theory of superconductivity, in Collected Papers of L. D. Landau, edited by D. ter Haar, Pergamon, New York, 1965, pp.546-568.

[12] L. P. Gorkov, Microscopic derivation of the Ginzburg-Landau equations in the theory of superconductivity, Sov. Phys. JETP 9(1959), 1364-1367.

[13] V. Grösch, K. Jansen, J. Jersák, C. B. Lang, T. Neuhaus, C. Rebbi, Monopoles and Dirac sheets in compact $U(1)$ lattice gauge theory, Phys. Lett. B 162(1985), 171-175.

[14] S. Grunewald, E.-M. Ilgenfritz, M. Müller-Preussker, Lattice vortices in the two-dimensional abelian Higgs model, Z. Phys. C-Particles and Fields 33(1987), 561-568.

[15] L. JACoBs, C. ReBBI, Interaction energy of superconducting vortices, Phys. Rev. B(3) 19(1978), 4486-4494.

[16] P. M. MArcus, Exact solution of the Ginzburg-Landau equations for slabs in tangential magnetic fields, Rev. Modern Phys. 36(1964), 294-299.

[17] F. Odeh, Existence and bifurcation theorems for the Ginzburg-Landau equations, J. Math. Phys. 8(1967), 2351-2356.

[18] C. ReBBI, Interaction of superconducting vortices, in Geom. Topol. Methods in Gauge Theory, Lecture Notes in Phys. vol. 129, Springer, Berlin, 1980, pp.96-113.

[19] V. V. Shмidт, Third critical field of superconductors with a film on the surface, in Physics and Metallurgy of Superconductors, edited by E. M. Savitskii and V. V. Baron, Plenum, New York, 1970 , pp.105-113.

[20] Y. YANG, The Ginzburg-Landau equations for superconducting films and the Meissner effect, preprint, 1988. 
Yisong Yang Existence, Regularity, and Asymptotic Behavior of the Solutions to the Ginzburg-Landau Equations on $\mathbf{R}^{3}$

Chjan. C. Lim On Symplectic Tree Graphs

Wilhelm I. Fushchich, Ivan Krivsky and Vladimir Simulik, On Vector and Pseudovector Lagrangians for Electromagnetic Field

Wilhelm I. Fushchich, Exact Solutions of Multidimensional Nonlinear Dirac's and Schrödinger's Equations

Wilhelm I. Fushchich and Renat Zhdanov, On Some New Exact Solutions of Nonlinear D'Allembert and Hamilton Equations

Brian A. Coomes, The Lorenz System Does Not Have a Polynomial Flow

J.W. Helton and N.J. Young, Approximation of Hankel Operators: Truncation Error in an $H^{\infty}$ Design Method

Gregory Ammar and Paul Gader, A Variant of the Gohberg-Semencul Formula Involving Circulant Matrices

R.L. Fosdick and G.P. MacSithigh, Minimization in Nonlinear Elasticity Theory for Bodies Reinforced with Inextensible Cords

Fernando Reitich, Rapidly Stretching Plastic Jets: The Linearized Problem

Francisco Bernis and Avner Friedman, Higher Order Nonlinear Degenerate Parabolic Equations

Xinfu Chen and Avner Friedman, Maxwell's Equations in a Periodic Structure

Avner Friedman and Michael Vogelius Determining Cracks by Boundary Measurements

Yuji Kodama and John Gibbons, A Method for Solving the Dispersionless KP Hierarchy and its Exact Solutions II

Yuji Kodama, Exact Solutions of Hydrodynamic Type Equations Having Infinitely Many Conserved Densities

Robert Carroll, Some Forced Nonlinear Equations and the Time Evolution of Spectral Data

Chjan. C. Lim Spanning Binary Trees, Symplectic Matrices, and Canonical Transformations for Classical N-body Problems

E.F. Assmus, Jr. and J.D. Key, Translation Planes and Derivation Sets

Matthew Witten, Mathematical Modeling and Computer Simulation of the Aging-Cancer Interface

Matthew Witten and Caleb E. Finch, Re-Examining The Gompertzian Model of Aging

Bei Hu, A Free Boundary Problem for a Hamilton-Jacobi Equation Arising in Ions Etching

T.C. Hu, Victor Klee and David Larman, Optimization of Globally Convex Functions

Pierre Goossens, Shellings of Tilings

D. David, D. D. Holm, and M.V. Tratnik, Integrable and Chaotic Polarization Dynamics in Nonlinear Optical Beams

D. David, D.D. Holm and M.V. Tratnik, Horseshoe Chaos in a Periodically Perturbed Polarized Optical Beam

Laurent Habsieger, Linear Recurrent Sequences and Irrationality Measures

Laurent Habsieger, MacDonald Conjectures and The Selberg Integral

David Kinderlehrer and Giorgio Vergara-Caffarelli, The Relaxation of Functionals with Surface Energies

Richard James and David Kinderlehrer, Theory of Diffusionless Phase Transitions

David Kinderlehrer, Recent Developments in Liquid Crystal Theory

Niky Kamran and Peter J. Olver, Equivalence of Higher Order Lagrangians

1. Formulation and Reduction

Lucas Hsu, Niky Kamran and Peter J. Olver, Equivalence of Higher Order Lagrangians II. The Cartan Form for Particle Lagrangians

D.J. Kaup and Peter J. Olver, Quantization of BiHamiltonian Systems

Metin Arik, Fahrünisa Neyzi, Yavuz Nutku, Peter J. Olver and John M. Verosky Multi-Hamiltonian Structure of the Born-Infeld Equation

David H. Wagner, Detonation Waves and Deflagration Waves in the One Dimensional ZND Model for High Mach Number Combustion

Jerrold R. Griggs and Daniel J. Kleitman, Minimum Cutsets for an Element of a Boolean Lattice

Dieter Jungnickel, On Affine Difference Sets

Pierre Leroux, Reduced Matrices and q-log Concavity Properties of q-Stirling Numbers

A. Narain and Y. Kizilyalli, The Flow of Pure Vapor Undergoing Film Condensation Between Parallel Plates

Donald A. French, On the Convergence of Finite Element Approximations of a Relaxed Variational Problem 
Author/s

Recent IMA Preprints (Continued) Title

Yisong Yang, Computation, Dimensionality, and Zero Dissipation Limit of the Ginzburg-Landau Wave Equation

Jürgen Sprekels, One-Dimensional Thermomechanical Phase Transitions with Non-Convex Potentials of Ginzburg-Landau Type

Yisong Yang, A Note On Nonabelian Vortices

Yisong Yang, On the Abelian Higgs Models with Sources

Chjan. C. Lim, Existence of Kam Tori in the Phase Space of Vortex Systems

John Weiss, Bäcklund Transformations and the Painlevé Property

Pu Fu-cho and D.H. Sattinger, The Yang-Baxter Equation for Integrable Systems

E. Bruce Pitman and David G. Schaeffer, Instability and Ill-Posedness in Granular Flow

Brian A. Coomes, Polynomial Flows on $\mathbb{C}^{n *}$

Bernardo Cockburn, Suchung Hou and Chi-Wang Shu, The Runge-Kutta Local Projection Discontinuous Galerkin Finite Element Method for Conservation Laws IV: The Multidimensional Case

Peter J. Olver, Invariant Theory, Equivalence Problems, and the Calculus of Variations

Daniel D. Joseph and Thomas S. Lundgren with an appendix by R. Jackson and D.A. Saville, Ensemble Averaged and Mixture Theory Equations

P. Singh, Ph. Caussignac, A. Fortes, D.D. Joseph and T. Lundgren, Stability of Periodic Arrays of Cylinders Across the Stream by Direct Simulation

Daniel D. Joseph, Generalization of the Foscolo-Gibilaro Analysis of Dynamic Waves

A. Narain and D.D. Joseph, Note on the Balance of Energy at a Phase Change Interface

Daniel D. Joseph, Remarks on inertial radii, persistent normal stresses, secondary motions, and non-elastic extensional viscosities

D. D. Joseph, Mathematical Problems Associated with the Elasticity of Liquids

Henry C. Simpson and Scott J. Spector, Some Necessary Conditions at an Internal Boundary for Minimizers in Finite Elasticity

Peter Gritzmann and Victor Klee, On the 0-1 Maximization of Positive Definite Quadratic Forms

Fu-Cho Pu and D.H. Sattinger, The Yang-Baxter Equations and Differential Identities

Avner Friedman and Fernando Reitich, A Hyperbolic Inverse Problem Arising in the Evolution of Combustion Aerosol

E.G. Kalnins, Raphael D. Levine and Willand Miller, Jr., Conformal Symmetries and Generalized Recurrences for Heat and Schrödinger Equations in One Spatial Dimension

Wang Jinghua and Gerald Warnecke, On Entropy Consistency of Large Time Step Godunov and Glimm Schemes

C. Guillopé and J.C. Saut, Existence Results for the Flow of Viscoelastic Fluids with a Differential Constitutive Law

H.L. Bodlaender, P. Gritzmann, V. Klee and J. Van Leeuwen Computational Complexity of Norm-Maximization

Li Ta-tsien (Li Da-qian) and Yu Xin, Life-Span of Classical Solutions to Fully Nonlinear Wave Equations

Jong-Shenq Guo, A Variational Inequality Associated with a Lubrication Problem

Jong-Shenq Guo, On the Semilinear Elliptic Equation $\Delta w-\frac{1}{2} y \cdot \nabla w+\lambda w-w^{-\beta}$ in $R^{n}$

Andrew E. Yagle, Inversion of the Bloch transform in magnetic resonance imaging using asymmetric two-component inverse scattering

Bei Hu, A Fiber Tapering Problem

Peter J. Olver, Canonical Variables for BiHamiltonian Systems

Michael Renardy, A Well-Posed Boundary Value Problem for Supercritical Flow of Viscoelastic Fluids of Maxwell Type

Michael Renardy, Ill-Posedness Resulting from Slip As a Possible Explanation of Melt Fracture

Michael Renardy, Compatibility Conditions at Corners Between Walls and Inflow Boundaries for Fluids of Maxwell Type

Rolf Rees, The Spectrum of Restricted Resolvable Designs with $r=2$

D. Lewis and J.C. Simo, Nonlinear stability of rotating pseudo-rigid bodies

Robert Hardt and David Kinderlehrer, Variational Principles with Linear Growth

San Yih Lin and Yisong Yang, Computation of Superconductivity in Thin Films

A. Narain, Pressure Driven Flow of Pure Vapor Undergoing Laminar Film Condensation Between Parallel Plates

P.J. Vassiliou, On Local Equivalence for Vector Field Systems 